\title{
Surface functionalization of epitaxial graphene on SiC by ion irradiation for gas sensing application
}

Priya Darshni Kaushik, Ivan Gueorguiev Ivanov, Pin-Cheng Lin, Gurpreet Kaur, Jens Eriksson, G. B. V. S. Lakshmi, D. K. Avasthi, Vinay Gupta, Anver Aziz, Azher M. Siddiqui, Mikael Syväjärvi and Gholamreza Yazdi

\section{Journal Article}

\section{Tweet}

N.B.: When citing this work, cite the original article.

Original Publication:

Priya Darshni Kaushik, Ivan Gueorguiev Ivanov, Pin-Cheng Lin, Gurpreet Kaur, Jens Eriksson, G. B. V. S. Lakshmi, D. K. Avasthi, Vinay Gupta, Anver Aziz, Azher M. Siddiqui, Mikael Syväjärvi and Gholamreza Yazdi, Surface functionalization of epitaxial graphene on SiC by ion irradiation for gas sensing application, Applied Surface Science, 2017. 403, pp.707-716. http://dx.doi.org/10.1016/j.apsusc.2017.01.111

Copyright: Elsevier

http://www.elsevier.com/

Postprint available at: Linköping University Electronic Press

http://urn.kb.se/resolve?urn=urn:nbn:se:liu:diva-136854

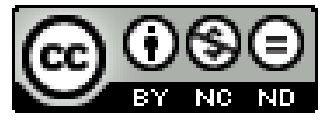




\title{
Surface functionalization of epitaxial graphene on SiC by ion irradiation for gas sensing application
}

Priya Darshni Kaushik ${ }^{1,2^{*}}$, Ivan G. Ivanov ${ }^{1}$, Pin-Cheng Lin $^{1}$, Gurpreet Kaur ${ }^{3}$, Jens Eriksson ${ }^{1}$, G. B. V. S. Lakshmi ${ }^{4}$, D. K. Avasthi ${ }^{4}$, Vinay Gupta ${ }^{3}$, Anver Aziz ${ }^{2}$, Azher M. Siddiqui ${ }^{2}$, Mikael Syväjärvi ${ }^{1}$, G. Reza Yazdi ${ }^{1 *}$

${ }^{1}$ Department of Physics, Chemistry and Biology, Linköping University, SE-58183 Linköping, Sweden.

${ }^{2}$ Department of Physics, Jamia Millia Islamia, New Delhi-110025 India

${ }^{3}$ Department of Physics and Astrophysics, University of Delhi, Delhi 110007, India

${ }^{4}$ Inter-University Accelerator Centre, Aruna Asaf Ali Marg, New Delhi 110067, India

*Corresponding authors: yazdi@ifm.liu.se, Tel: +46-13-282544, Fax: +46-13-137568, kaushik.priyadarshni@gmail.com

\begin{abstract}
:
In this work surface functionalization of epitaxial graphene grown on silicon carbide was performed by ion irradiation to investigate their gas sensing capabilities. Swift heavy irradiation using $100 \mathrm{MeV}$ silver ions at four varying fluences was implemented on epitaxial graphene to investigate morphological and structural changes and their effects on the gas sensing capabilities of graphene. Sensing devices are expected as one of the first electronic applications using graphene and most of them use functionalized surfaces to tailor a certain function. In our case we have studied irradiation as a tool to achieve functionalization. Morphological and structural changes on epitaxial graphene layers were investigated by atomic force microscopy, Raman spectroscopy, Raman mapping and reflectance mapping. The surface morphology of irradiated graphene layers showed graphene folding, hillocks, and formation of wrinkles at highest fluence $\left(2 \times 10^{13}\right.$ ions $\left./ \mathrm{cm}^{2}\right)$. Raman spectra analysis shows that the graphene defect density is increased with increasing fluence, while Raman mapping and reflectance mapping show that there is also a reduction of monolayer graphene coverage. The samples were investigated for ammonia and nitrogen dioxide gas sensing applications. Sensors fabricated on pristine and irradiated samples showed highest gas sensing response at an optimal fluence. Our work provides new pathways for introducing defects in controlled manner in epitaxial graphene, which can be used not only for gas sensing application but also for other applications, such as electrochemical, biosensing, magnetosensing and spintronic applications.
\end{abstract}


Keywords: Epitaxial graphene, Surface functionalization, Ion irradiation, gas sensors

\section{Introduction:}

Graphene with two-dimensional honeycomb lattice structure of $\mathrm{sp}^{2}$-bonded carbon atoms acts as excellent candidate for future nano electronics due to its outstanding properties such as longrange ballistic transport at room temperature, optical transparency, higher carrier mobility, electrical and thermal conductivity properties [1-3]. Graphene can be fabricated by different methods such as mechanical exfoliation of graphite [4], chemical exfoliation [5], chemical vapour deposition (CVD) [6] and epitaxial graphene (EG) grown on silicon carbide (SiC) [7]. Amongst all these techniques, growth of $\mathrm{EG}$ on $\mathrm{SiC}$ has several advantages. The wide band gap semiconductor $\mathrm{SiC}$ acts as a suitable substrate for not only various industrial applications but also provides wafer scale growth of epitaxial graphene [7]. On the contrary, graphene fabricated using chemical exfoliation technique suffers from lots of defects due to metallic impurities which alter electronic, electrochemical and redox properties of graphene [8]. Additionally, graphene grown using CVD technique on metal substrate requires transferring onto an insulating substrate for its industrial use and to date an efficient transfer process is not available [6]. Epitaxial graphene grown on $\mathrm{SiC}$ has fewer defects and does not require any additional transfer to another substrate. Hence, epitaxially grown graphene is free from processing pollutant, which alters electrical, mechanical and sensing property of graphene $[9,10]$. Covalent functionalization and defects like vacancy defects, substitutional defects and topological defects introduced in controlled manner on the graphene surface, can further tailor and improve its electronic, sensing and magnetic properties. Functionalization of EG layer with polymers which create a type of $\mathrm{sp}^{3}$ defects enhances electronic application of epitaxial graphene [11]. Doping of graphene with nitrogen introduces substitutional defects and provide a way for tailoring its electronic and chemical properties [12]. In addition, decorating EG with metals like Au and Pt increases its gas sensing ability [13]. Further band gap engineering and velocity renormalization is achieved by depositing Ti layer on graphene [14], while improved transistor performance is obtained by integration of EG with dielectric materials [15]. Graphene has also the ability of reconstructing its hexagonal carbon lattice into pentagons and heptagons near the defect sites under electronic beam irradiation [16]. These defects act as a tool for manipulating physical properties of graphene and result in device-based applications of graphene, such as FETs [17], RF transistors and amplifiers [18], sensors [19], detectors [20] and metrology [21]. 
Ion beam technology acts as a perfect tool to introduce defects in graphene due to its high uniformity, repeatability, single sided doping capability and ability to change the surface without affecting the bulk material. Previously, low energy ion implantation has been used to dope graphene using $50 \mathrm{eV}$ nitrogen ions at low temperature [22]. Swift heavy ion (SHI) irradiation is the process of irradiating materials with energy in the range of MeV. In SHI irradiation, electronic energy loss dominates over nuclear energy loss, contrary to nuclear energy loss domination during low energy ion implantation [23]. Previously, SHI irradiation has been used to study structural and electrical changes in graphene [24-26]. Kumar et al used silver ion irradiation with $100 \mathrm{MeV}$ energy for graphene layer grown by CVD. They observed that up to $3 \times 10^{11}$ ions $/ \mathrm{cm}^{2}$ fluence the defect density decreased compared to pristine sample as the annealing process reduced damage that occurred due to the irradiation [24]. However at fluence greater than $3 \times 10^{11}$ ions $/ \mathrm{cm}^{2}$, the defect density increased due to domination of damages over the restoring action of the annealing process [24]. Change from n-doped to p-doped layer in single layer graphene was observed by Ochedowski et al following irradiation at glancing angle of incidence with $92 \mathrm{MeV}$ energy [25]. Further, unzipping and folding of exfoliated graphene using SHI has been reported [26].

However, despite high reproducibility and wider industrial applications of EG on SiC, SHI irradiation has never been employed to study structural changes in EG and subsequently investigate its applicability for sensing use. Since SHI is a powerful tool for altering the surface of graphene, in this work we investigated different types of defect generation in EG grown on $4 \mathrm{H}-\mathrm{SiC}$ substrate and its application for gas sensing. Since we were interested to study defects in EG, we had chosen the fluence range from $6.6 \times 10^{11}$ ions $/ \mathrm{cm}^{2}$ to $2 \times 10^{13} \mathrm{ions} / \mathrm{cm}^{2}$ in consideration of a previous study showing decrease in defect density up to $3.3 \times 10^{11} \mathrm{ions} / \mathrm{cm}^{2}$ in CVD grown graphene following $100 \mathrm{MeV}$ SHI [24].

By studying irradiation induced defects in graphene, our ultimate aim is to control gas sensing ability of the irradiated samples. Gas sensing capabilities of epitaxial graphene in ppm and ppb range have been reported in the past [19, 27]. Defects like vacancy and substitutional defects create preferential bonding sites for adsorption of atoms and molecules, and can be exploited for enhancing gas and liquid sensing application of graphene [28]. Epitaxial graphene shows gas sensing due to physio-adsorption of gas molecules whereas defective graphene forms a bond with the gas molecules thereby resulting in increased sensing ability of graphene [29]. A theoretical study by Zhang et al showed that sensitivity and selectivity of graphene based gas sensors can be significantly improved by introducing defects in graphene [30]. In our work, the 
gas sensing application of SHI irradiated epitaxial graphene on $\mathrm{SiC}$ is explored and reported for the first time to our knowledge.

In this study, Atomic Force Microscopy (AFM) and Raman spectroscopy showed that generation of various defects in epitaxial graphene depended on fluence of heavy ion irradiation. AFM images revealed hillocks and folding of single layer graphene due to formation of array of defects on graphene layer surface and SiC surface. Moreover, we have observed formation of wrinkles in the graphene at higher fluence. The gas sensing ability of EG was observed to increase with introduction of defects with SHI. Our work provides a new source for introducing and controlling defects in EG on SiC, which can be used not only for gas sensing application but also for other applications like electrochemical, biosensing, magnetosensing and spintronic applications.

\section{Experimental}

Growth of epitaxial graphene was performed on $4 \mathrm{H}-\mathrm{SiC}(0001)$ at $2000^{\circ} \mathrm{C}$ and $1 \mathrm{~atm}$ argon pressure in an inductively heated furnace as described previously [31]. Swift ion irradiation on EG samples was done using 15 MV Pelletron Accelerator Facility (at IUAC, New Delhi, India). Silver ions with $100 \mathrm{MeV}$ at $6.6 \times 10^{11}, 2 \times 10^{12}, 6.6 \times 10^{12}$ and $2 \times 10^{13}$ ions/ $\mathrm{cm}^{2}$ fluence were used for irradiation. The pressure in the irradiation chamber was of the order of $10^{-6}$ mbar and the average current was 2-particle nano ampere. The projected range, electron and nuclear energy loss were calculated using Stopping and Range of Ions in Matter (SRIM) software [32]. This calculation showed that the projected range of stable $100 \mathrm{MeV}$ silver ion into graphene on SiC is $13.9 \mu \mathrm{m}$ with nuclear energy loss $\left(\mathrm{S}_{\mathrm{n}}\right)$ to be $0.0586 \mathrm{keV} / \mathrm{nm}$ and electron energy loss $\left(\mathrm{S}_{\mathrm{e}}\right)$ to be $11.9 \mathrm{keV} / \mathrm{nm}$ resulting in $\mathrm{S}_{\mathrm{e}} / \mathrm{S}_{\mathrm{n}}$ ratio of 203.

Atomic force microscopy in topological and phase mode have been used to study the graphene surface morphology and thickness uniformity. The AFM was performed using a Dimension 3100 AFM (Digital Instruments, Veeco, CA) operated in tapping mode. Fluence dependent damages in graphene were analyzed using Raman spectra taken with $488 \mathrm{~nm}$ laser excitation using Jobin Yvon Horiba Labram HR microscope with spot size of around $1 \mu \mathrm{m}$. Raman mapping was done with micro Raman set up with excitation laser of $532 \mathrm{~nm}$ and spatial resolution of $<1 \mu \mathrm{m}$ to obtain detailed information about the defects generated after irradiation and also to determine the homogeneity of the defect distribution. To determine the number of graphene layers folded during irradiation, reflectance mapping was performed using micro 
Raman spectrometer with the configuration described in reference [33]. Reflectance mapping is based on mapping the power of laser beam reflected from sample in micro Raman set up and can efficiently determine more than 10 graphene layers which is not possible by AFM [33]. However, due to limited resolution of reflectance mapping in comparison with AFM that has higher resolution in nanometer range, percentage of monolayer and bilayer graphene were calculated using AFM [33].

For gas sensing, firstly Ti/Au metal contacts were fabricated by magnetron sputtering on the irradiated graphene layer on $\mathrm{SiC}$ substrates. The as-prepared samples were glued onto TO8 16-pin headers in order to perform the gas testing. The current/voltage signals were transmitted from the metal contacts to the pins on the header. The gas testing was conducted with an in-house gas mixing systems and all measurements were done at room temperature. A Keithley 2100 Sourcemeter was used to measure the resistance. Before the testing, the sensors were flushed by a background gas composed of $80 \%$ nitrogen and $20 \%$ oxygen in order to stabilize the sensor baseline. The total flow rate was fixed at $100 \mathrm{ml} / \mathrm{min}$ and the same stabilizing method was used for the sensors between every gas exposure. Gas sensing capability of the irradiated samples was investigated for ammonia $\left(\mathrm{NH}_{3}\right)$ and nitrogen dioxide $\left(\mathrm{NO}_{2}\right)$. Sensors fabricated on pristine and irradiated samples at $6.6 \times 10^{11}$ ions $/ \mathrm{cm}^{2}, 2 \times 10^{12} \mathrm{ions} / \mathrm{cm}^{2}$ and $6.6 \times 10^{12}$ ions $/ \mathrm{cm}^{2}$ fluences were exposed at room temperature to $\mathrm{NH}_{3}$ gas for $1 \mathrm{~h}$ at $10 \mathrm{ppm}$ and 50 ppm concentrations; and to $\mathrm{NO}_{2}$ gas for $30 \mathrm{~min}$ at 10- and 50- ppm. A UV-LED of wavelength $365 \mathrm{~nm}$ was used for desorption of $\mathrm{NO}_{2}$.

\section{Results and Discussion}

Topological AFM analysis represent how surface morphology changes with the experimental conditions. Reflectance maps and AFM images in Fig. 1 show the thickness uniformity and surface morphology of EG on SiC substrate before and after swift heavy ion irradiation with silver ions at $2 \times 10^{13}$ ions $/ \mathrm{cm}^{2}$ fluence. Fig. 1(a) and (b) show reflectance maps of pristine and highest irradiated graphene, respectively on SiC over a $30 \mu \mathrm{m} \times 30 \mu \mathrm{m}$ area. For pristine sample (Fig. 1a) 99\% of the surface covered by ML and bilayer graphene, and for irradiated sample (Fig. 1b) $26 \%$ covered by ML and bilayer graphene and the rest is multilayer graphene. The AFM images in Fig. 1(c) and (d) show the surface topography of graphene layer before and after irradiation on the same samples used for reflectance maps (parts a and b in Fig. 1). Fig. 1(d) shows hillocks, wrinkles and folding due to formation of multilayer graphene which can be 
observed as change in contrast in comparison to pristine sample. Distinguishing different graphene layers based on change in contrast of AFM phase images has been previously reported $[34,35]$. Based on AFM phase image (not shown) on pristine sample coverage of ML and bilayer graphene are 95\% and 5\%, respectively. Fig. 1(e) and (f) show higher magnification of the area in Fig. 1(c) and Fig. 1(d), respectively. Fig 1(f) shows hillocks, wrinkles and folding on surface of EG on SiC substrate.

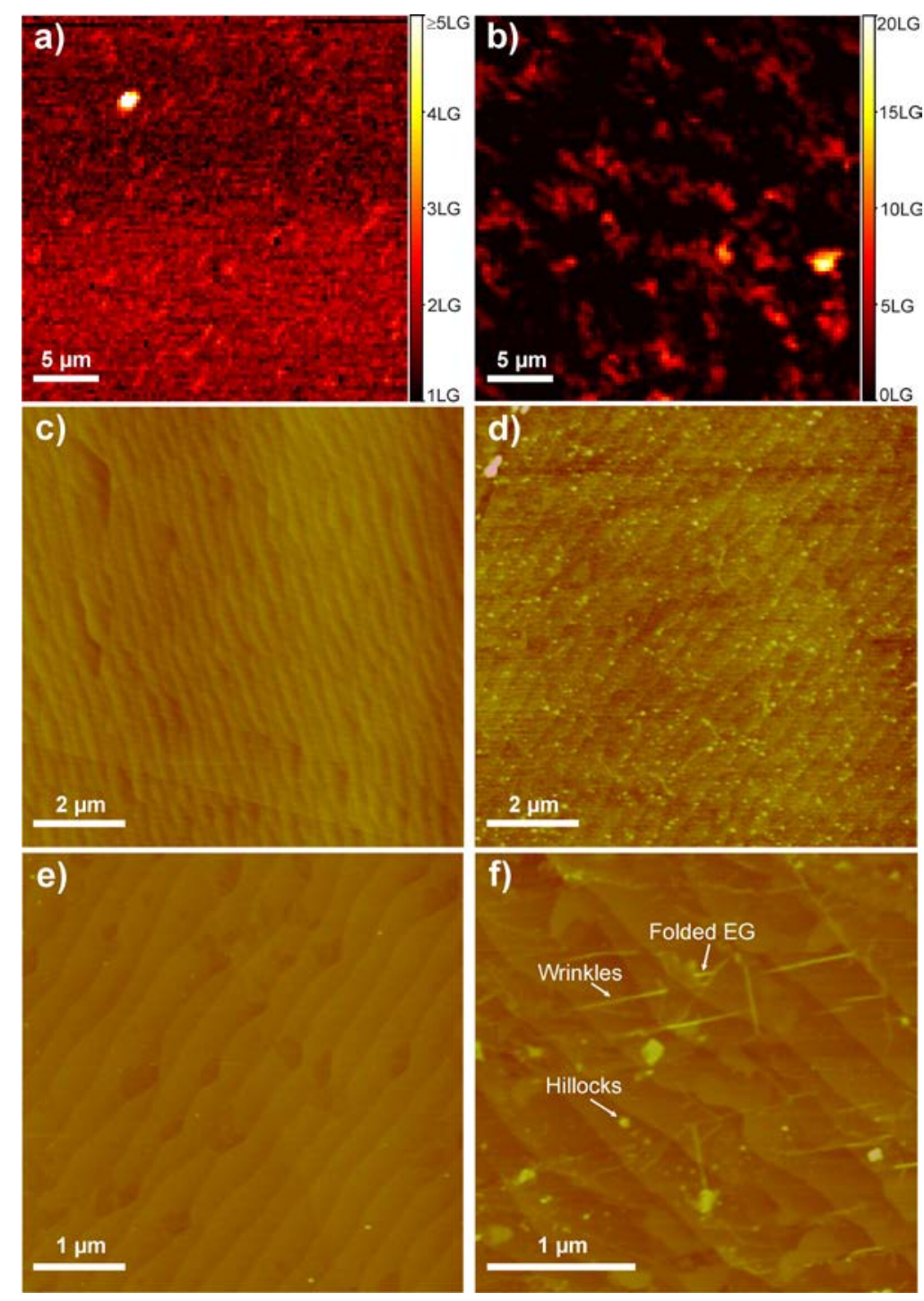

Fig. 1. Reflectance map and AFM images of pristine and irradiated graphene. Reflectance map of (a) pristine, (b) irradiated graphene layer. The white spot in map (a) indicates multilayer graphene, most probably due to micropipe in the substrate. Topological AFM images of (c) pristine, and (d) irradiated graphene layer. (e) and (f) present higher magnification images from the areas in parts (c) and (d), respectively. 
Zhao et al reported that rupturing of supported graphene requires energy $\mathrm{S}_{\mathrm{e}}$ to be greater than the threshold of $6.5 \mathrm{keV} / \mathrm{nm}$ [36]. In this study, the provided $\mathrm{S}_{\mathrm{e}}$ was $11.9 \mathrm{keV} / \mathrm{nm}$, and the graphene layers were ruptured. This resulted in folding of ML and bilayer graphene and consequently converting them to multi-layer graphene randomly distributed on the substrate. For the sample exposed to the highest irradiation, ruptured graphene layers are visible in the AFM images (Fig. 1(d) and Fig. 1(f)), as well as in the reflectance map, Fig. 1(b).

In SHI, when a fast moving ion passes along a straight-line trajectory perpendicular to the surface plane, an extremely localized energy release may lead to creation of single nanosized hillocks on the surface. Hillock formation has been reported previously [37-39] and their formation is explained by the thermal spike model [40] in which high-energy ion bombardment gives rise to a cylindrically shaped ion track of nanometric dimension along the ion path. This energy is transferred by electron - phonon coupling to the atomic system and causes localized heating along the ion track which results in transient thermal spikes that last for few picoseconds and gives rise to nanosized hillocks at the impact point of each ion [41]. Similar observation was reported earlier for mechanically exfoliated graphene following $\sim 100 \mathrm{MeV}$ SHI [26]. Additionally, we also observed wrinkles with non-uniform height, and mainly elongated in the $(1 \overline{2} 0)$ direction of the substrate, as shown in Fig. 1(f).

To determine fluence dependent defect formation on graphene surface, AFM study was done over scan areas of $1 \times 1 \mu \mathrm{m}$ (Fig. 2) on samples irradiated at fluences $6.6 \times 10^{11}, 2 \times 10^{12}$, $6.6 \times 10^{12}$ and $2 \times 10^{13}$ ions $/ \mathrm{cm}^{2}$. Figs. $2(\mathrm{a}-\mathrm{c}$ ) show some structural defects which may represent the initial stage of formation of folding on the surface of graphene whose intensity increases with increasing the fluence. Surface roughness increased with increasing fluence, and the measured value using AFM for pristine and highest irradiated sample $\left(2 \times 10^{13}\right.$ ions $\left./ \mathrm{cm}^{2}\right)$ are about $0.27 \mathrm{~nm}$ and $1 \mathrm{~nm}$, respectively. This increasing in roughness with increasing defects density agrees with previous report [42].

In the sample subjected to the highest fluence we can clearly observe wrinkles (Fig. 1(f) and 2(d)). Wrinkles are formed during the cooling process as a result of releasing compressive strain on graphene layers induced by thermal mismatch between the epitaxial graphene layer and SiC substrate [43]. At highest fluence $\left(2 \times 10^{13} \mathrm{ions} / \mathrm{cm}^{2}\right)$ wrinkles are observed due to higher 
temperature generated at higher fluence resulting in higher compressive strain, which leads to formation of wrinkles on the graphene surface [44].
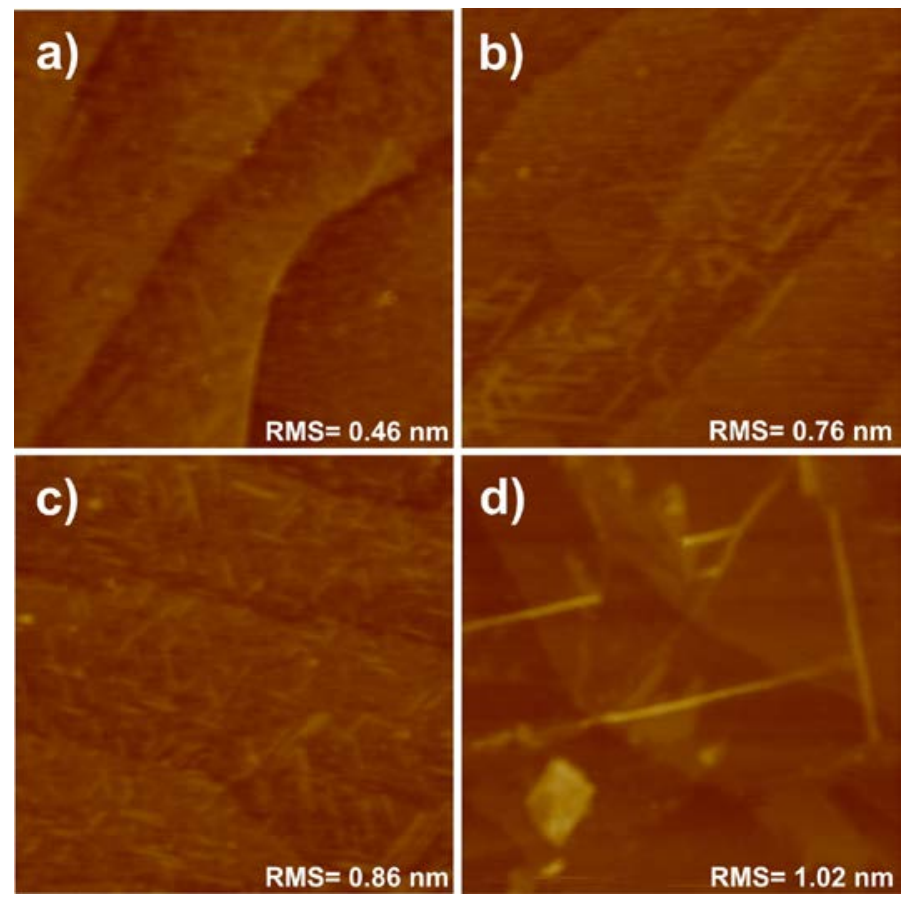

Fig. 2. AFM height images of irradiated graphene samples, at (a) $2 \times 10^{11} \mathrm{ions} / \mathrm{cm}^{2}$ (b) $2 \times 10^{12} \mathrm{ions} / \mathrm{cm}^{2}$ (c) $6.6 \times 10^{12} \mathrm{ions} / \mathrm{cm}^{2}$ and (d) $2 \times 10^{13}$ ions $/ \mathrm{cm}^{2}$ fluences. Scan size $1 \mu \mathrm{m} \times 1 \mu \mathrm{m}$.

Besides surface roughness, structural defects, hillocks and wrinkles, we also observed folding of graphene at all fluences. Fig. 3(a) shows graphene folding at $2 \times 10^{12} \mathrm{ions} / \mathrm{cm}^{2}$ over a scan area of $2 \times 2 \mu \mathrm{m}$ which is seen more clearly over a scan area of $700 \mathrm{~nm} \times 700 \mathrm{~nm}$ as shown in Fig. 3(b). Smith et al reported that the minimum incident electron energy required to knock out a carbon atom from the graphene lattice is $86 \mathrm{keV}$ [45]. Since an incident energy of 100 $\mathrm{MeV}$ was used in the current study, it is likely that carbon atoms were knocked out from the graphene lattice creating vacancy defects which further agglomerated into larger vacancy structures due to prolonged irradiation. A minimum energy of around 5-7 eV is required to form stable pentagon/heptagon defects in graphene from their regular hexagonal honeycomb like structure [46]. Additionally, Zhao et al reported that minimum $5 \mathrm{keV} / \mathrm{nm}$ electronic energy deposition is required to create other defects in graphene such as Stone-Wales defect, vacancy defects and local distorted carbon rings defects [36]. Since in this study, $S_{e}=11.9 \mathrm{keV} / \mathrm{nm}$, hence all such defects are likely to be present on the graphene surface. It has been shown previously that at glancing angle of incidence, folding is homogenous but at perpendicular angle 
of incidence folding is inhomogeneous [26]. Moreover, Akcöltekin et al reported that multiple but aligned defects are formed during SHI, resulting in reconstruction of the carbon network along the ion track. They reported that chains of hillocks coincide exactly with the line of extended defects and results in unzipping the graphene along the ion track [26]. Due to this unzipping, graphene gets folded at many places along the ion track, which is seen in Fig. 3. However, the folding is inhomogenous since the irradiation is done at normal incidence.
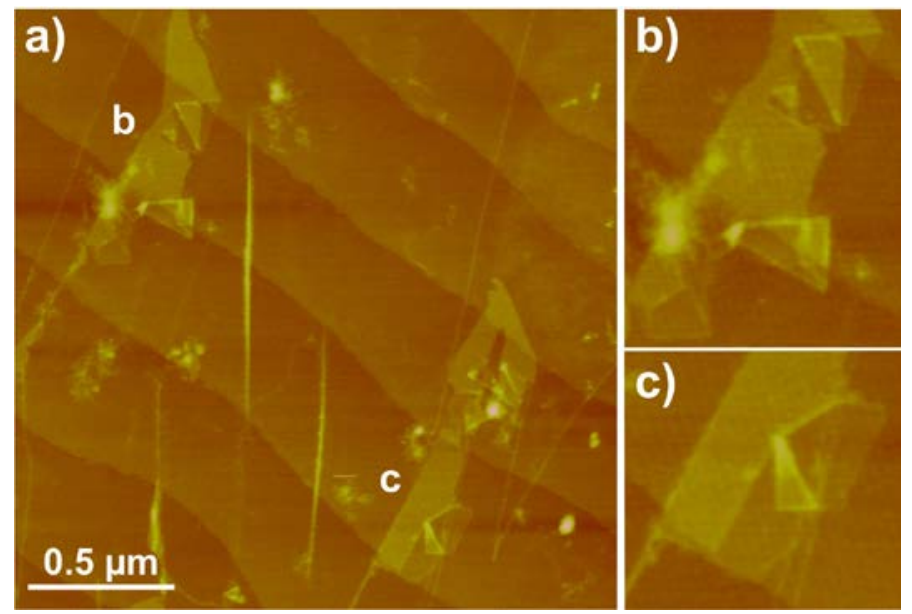

Fig. 3. AFM images of (a) folded graphene on $\mathrm{SiC}$ after irradiation at $2 \times 10^{12} \mathrm{ions} / \mathrm{cm}^{2}$ fluence (scan size $1 \mu \mathrm{m} \times 1 \mu \mathrm{m}$ ). (b) and (c) present higher magnification of points b and $\mathrm{c}$ in part (a).

Raman spectroscopy and reflectance mapping have been used to study the defect density and multiplicity of graphene folding on irradiated samples. Fig. 4(a-e) shows Raman spectra of pristine and irradiated graphene samples using a laser with excitation wavelength of 488nm. The spectra exhibit changes in D, G and 2D peak due to swift heavy ion irradiation. Monolayer pristine graphene is characterized by $\mathrm{G}$ peak around $1580 \mathrm{~cm}^{-1}$ and 2D peak around $2700 \mathrm{~cm}^{-1}[47]$. 


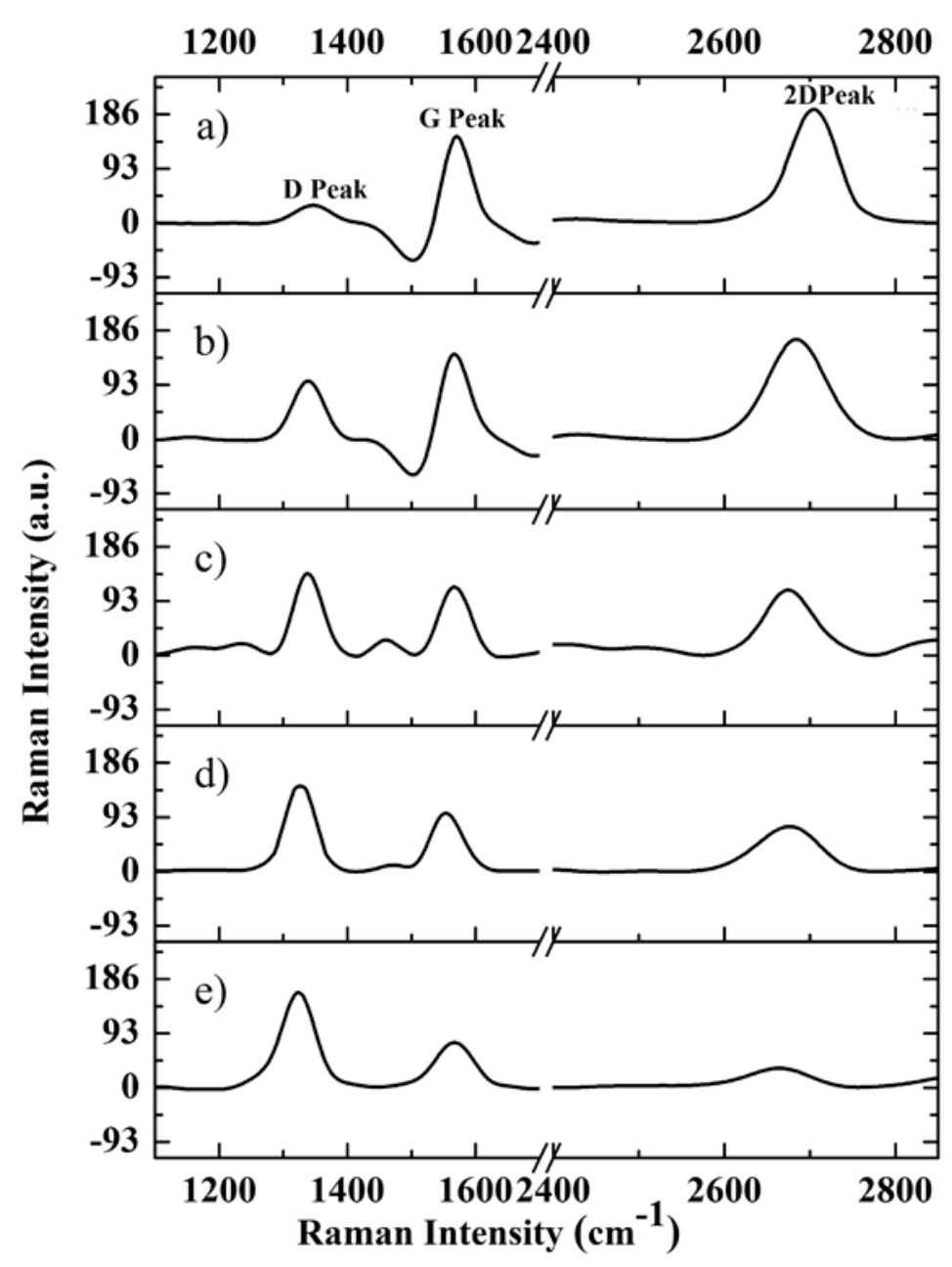

Fig. 4. Raman spectrum of (a) pristine, and irradiated graphene on $\mathrm{SiC}$ at (b) $2 \times 10^{11} \mathrm{ions} / \mathrm{cm}^{2}$, (c) $2 \times 10^{12}$ ions $/ \mathrm{cm}^{2}$, (d) $6.6 \times 10^{12}$ ions $/ \mathrm{cm}^{2}$ and (e) $2 \times 10^{13}$ ions $/ \mathrm{cm}^{2}$ fluences using a laser with excitation wavelength of $488 \mathrm{~nm}$. The structure of the left side of $\mathrm{G}$ peak for pristine graphene is due to the buffer layer and there is no $\mathrm{D}$ peak for pristine graphene.

The $G$ peak is associated with the $E_{2 g}$ phonon mode at Brillouzin zone center [48]. The breathing mode of six-atom rings gives rise to D peak around $1350 \mathrm{~cm}^{-1}$, but requires defects for its activation and its intensity increases with increasing defects. It arises from transverse optic phonons around the K point [49]. The D peak in the pristine sample (Fig. 4(a) and 5(a)) is actually buried in the contribution of the buffer layer, which confirms the high quality of epitaxial graphene. This was also confirmed by the reflectance and AFM phase images, as grown graphene on SiC substrate was dominated by monolayer with some bilayer formation. The second order of the $\mathrm{D}$ peak is the $2 \mathrm{D}$ peak, which is allowed even in absence of defects since it 
involves creation of two phonons with opposite momenta. Its intensity decreases with increase in defects [50]. The EG with single layer graphene is characterized by single Lorentzian 2D peak [47]. In case of bilayer, the 2D peak is broader and can be fitted by four Lorentzian peaks. However, the Raman spectrum of 5-layered graphene is hardly distinguishable from that of graphite [51]. Fig. 4 (b-e) shows that with increasing the ion fluence, the intensity around the D peak increases while that of the $G$ peak decreases. The ratio $I(D) / I(G)$, sometimes called disorder parameter in graphene, serves as parameter for estimating the defect density [24]. Here I(D) and I(G) denote the peak intensities of the D and G peaks, respectively, and are obtained after fitting the $D$ and $G$ peak with Lorentzian functions. We found $I(D) / I(G)$ to increase with increasing fluence from approximately zero in pristine sample to 2.055 in the highest irradiated sample $\left(2 \times 10^{13}\right.$ ions $\left./ \mathrm{cm}^{2}\right)$. Cancado et al suggested that defect formation in graphene can be divided into two stages on the base of $I(D) / I(G)$, with stage (i) and (ii) having low and high defect concentration, respectively [52]. In stage (i), I(D)/I(G) increases monotonically with increasing defect density and varies according to the following formula:

$$
\mathrm{I}(\mathrm{D}) / \mathrm{I}(\mathrm{G}) \propto 1 / \mathrm{L}_{\mathrm{D}}^{2} \text {, }
$$

where, $L_{D}$ is average distance between defects. On the other hand, in stage (ii) $I(D) / I(G)$ decreases according to the equation

$$
\mathrm{I}(\mathrm{D}) / \mathrm{I}(\mathrm{G}) \propto \mathrm{L}_{\mathrm{D}}{ }^{2} \text {. }
$$

The transition from stage (i) to stage (ii) is observed when the I(D)/I(G) ratio is around 3 and $\mathrm{L}_{\mathrm{D}}$ is in-between 2 to $5 \mathrm{~nm}$ using a $2.41 \mathrm{eV}(532 \mathrm{~nm})$ laser excitation. In the present work, $\mathrm{I}(\mathrm{D}) / \mathrm{I}(\mathrm{G})$ ratio increased to 2.055 at the highest fluence $\left(2 \times 10^{13} \mathrm{ions} / \mathrm{cm}^{2}\right)$ and no decrease was observed with increasing fluence, thereby indicating that at all fluences stage (i) defect generation should be considered. Thus, as expected, the defects increase with increase in fluence which is evident both by increase in roughness (observed by AFM) and increase in $I(D) / I(G)$ ratio. Fig. 4 shows that the intensity of the $2 \mathrm{D}$ peak with respect to the $\mathrm{G}$ peak decreases with increasing fluence, and also the FWHM of the 2D peak increases at higher fluences suggesting conversion of monolayer graphene to multilayer graphene [51, 53]. This was further supported by the AFM, which shows that folding clearly appears in the irradiated sample (Fig. 1 and Fig. 3), resulting in formation of multilayer graphene [35]. Besides folding, decrease in intensity of 2D peak with respect to $G$ peak could also be attributed to other factors such as doping. Ochedowski et al showed that SHI of exfoliated single layer graphene at $92 \mathrm{MeV}$ with xenon ions resulted in doping of graphene and thereby increasing charged impurities [54]. Further, Ni 
et al reported that increase in charge impurities results in decrease of $2 \mathrm{D}$ peak intensity with respect to the $G$ peak [55]. In the present study, decrease in the intensity of 2D peak with respect to $G$ peak upon irradiation maybe attributed to both conversion of monolayer graphene to multilayer graphene and increase of charge impurities due to doping upon SHI. Fig. 4 also shows a red shift in $2 \mathrm{D}$ peak intensity of the irradiated samples in comparison to that in the pristine sample. Such shift could be due to the presence of tensile strain as previously reported [56].

Zandiatashbar et al used $\mathrm{I}(\mathrm{D}) / \mathrm{I}(\mathrm{G})$ and $\mathrm{I}(2 \mathrm{D}) / \mathrm{I}(\mathrm{G})$ ratios to distinguish between sp ${ }^{3}$ defect and vacancy defects [57]. They reported that if $\mathrm{I}(\mathrm{D}) / \mathrm{I}(\mathrm{G})<1$ and $\mathrm{I}(2 \mathrm{D}) / \mathrm{I}(\mathrm{G})>1$, then it is a sp ${ }^{3}$ defect region. On the contrary if $\mathrm{I}(\mathrm{D}) / \mathrm{I}(\mathrm{G})>1$ and $\mathrm{I}(2 \mathrm{D}) / \mathrm{I}(\mathrm{G})<1$, then it is a vacancy defect region. Here $\mathrm{I}(2 \mathrm{D})$ represents peak intensity or the peak amplitude of the $2 \mathrm{D}$ peak which is obtained after fitting the 2D peak with Lorentzian function. In our study, at $6.6 \times 10^{11} \mathrm{ions} / \mathrm{cm}^{2}$ fluence, the $\mathrm{I}(\mathrm{D}) / \mathrm{I}(\mathrm{G})$ and $\mathrm{I}(2 \mathrm{D}) / \mathrm{I}(\mathrm{G})$ are 0.630 and 1.1 respectively, which is indicative of $\mathrm{sp}^{3}$ defect generation upon SHI. At higher fluences $\left(2 \times 10^{12} \mathrm{ions} / \mathrm{cm}^{2}, 6.6 \times 10^{12} \mathrm{ions} / \mathrm{cm}^{2}\right.$ and $2 \times 10^{13}$ ions $/ \mathrm{cm}^{2}$ ) the $\mathrm{I}(\mathrm{D}) / \mathrm{I}(\mathrm{G})$ ratio became greater than 1 and $\mathrm{I}(2 \mathrm{D}) / \mathrm{I}(\mathrm{G})$ became less than 1 . At $2 \times 10^{13}$ ions/cm² fluence, $\mathrm{I}(\mathrm{D}) / \mathrm{I}(\mathrm{G})$ and $\mathrm{I}(2 \mathrm{D}) / \mathrm{I}(\mathrm{G})$ reached a maximum and minimum value of 2.055 and 0.50 respectively, which suggests vacancy defect generation upon SHI at higher fluences.

Along with the prominent peaks (D, G and 2D), the Raman specta also show some additional peaks; D', D+D' and 2D' around 1620, $2950 \mathrm{~cm}^{-1}$ and $3240 \mathrm{~cm}^{-1}$ respectively. The $\mathrm{D}^{\prime}$ peak originate from intra-valley double resonance process in the presence of defects. The $\mathrm{D}+\mathrm{D}^{\prime}$ peak also requires defects for its activation and arises from two phonons with different momentum [58]. The second overtone of $\mathrm{D}^{\prime}$ is the $2 \mathrm{D}^{\prime}$ mode, which does not require defects for its activation but arises from the process where momentum conservation is satisfied with two phonons with opposite wave-vectors [59]. In order to investigate the presence of $\mathrm{D}^{\prime}, \mathrm{D}^{+} \mathrm{D}^{\prime}$ and 2D' peaks in Raman spectra, and to investigate the homogeneity of the irradiated samples, we did Raman mapping on samples irradiated at $2 \times 10^{12}$ ions $/ \mathrm{cm}^{2}$ and $2 \times 10^{13}$ ions $/ \mathrm{cm}^{2}$ fluences using $532 \mathrm{~nm}$ laser in backscattering geometry. Each map contains 256 Raman spectra ordered on a grid with $300 \mathrm{~nm}$ pitch enclosed in a 4.5 x $4.5 \mu \mathrm{m}$ square. The Raman spectrum obtained from pristine sample shows only the G, $2 \mathrm{D}$ and the $2 \mathrm{D}^{\prime}$ peaks with clear contribution from the buffer layer (Fig. 5(a)). However, in the irradiated samples two additional peaks appear along with the $\mathrm{D}$ peak, $\mathrm{D}^{\prime}$ and $\mathrm{D}+\mathrm{D}^{\prime}$ peaks, which are representative of significant defect concentrations in these samples (Fig. 5(b) -(c)) [58]. Raman mapping of the sample irradiated at $2 \times 10^{12}$ ions $/ \mathrm{cm}^{2}$ fluence showed broadening of the $2 \mathrm{D}$ peak only in a few spectra, while the broadening was more often found in the spectra of the highest irradiated sample (at $2 \times 10^{13}$ 
ions $\left./ \mathrm{cm}^{2}\right)$. In addition, many Raman spectra obtained from the maps of both fluences $\left(2 \times 10^{12}\right.$ ions $/ \mathrm{cm}^{2}$ and $2 \times 10^{13}$ ions $/ \mathrm{cm}^{2}$ ) show no contribution from graphene, indicating that in these regions the graphene and the underlying buffer layer has been removed from the substrate surface. The number of spectra with no graphene contribution is larger in the highest-fluence sample than the one at lower fluence.

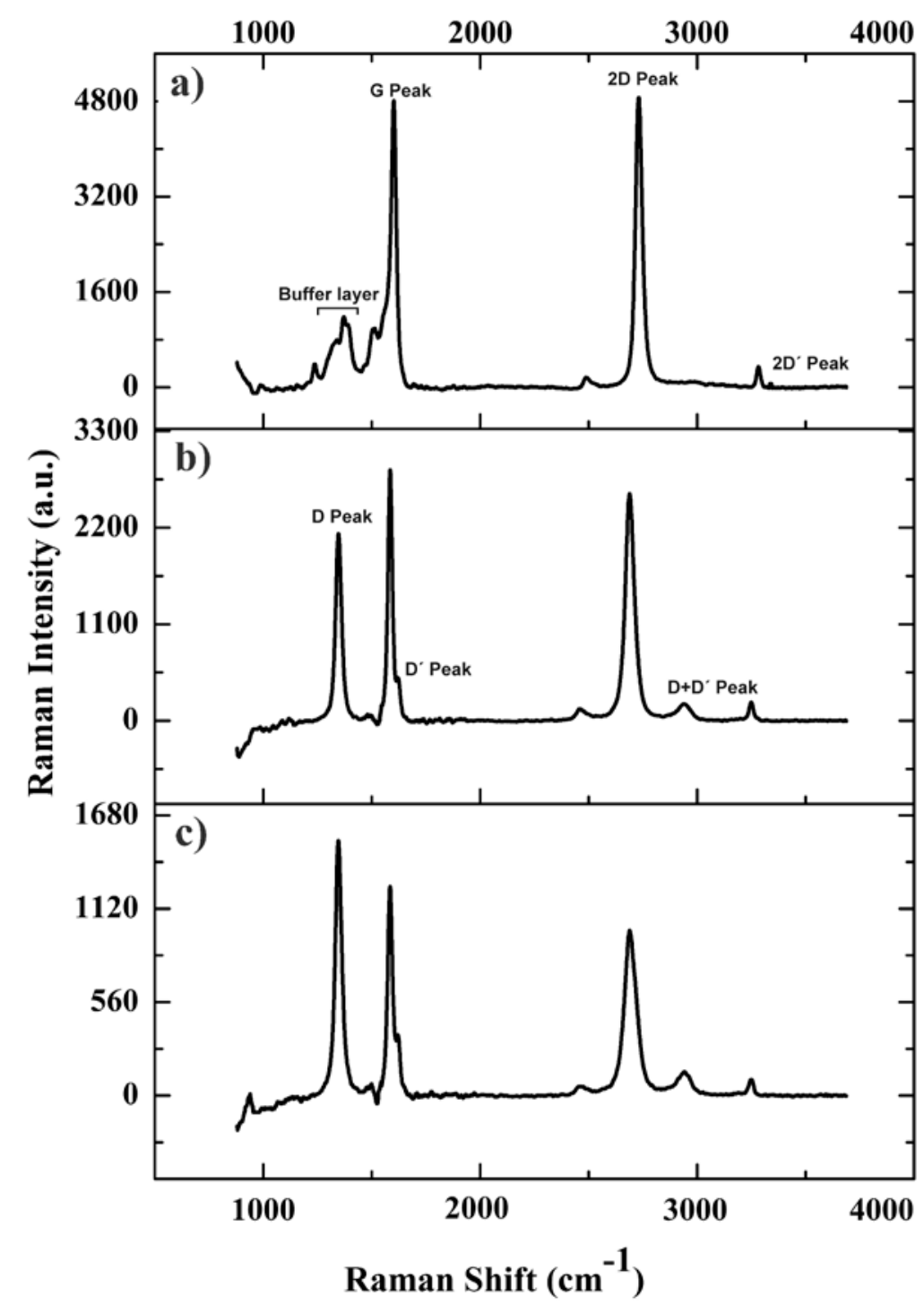

Fig. 5. Raman spectrum obtained from Raman Mapping of (a) pristine, and irradiated graphene samples at (b) $2 \times 10^{12}$ ions $/ \mathrm{cm}^{2}$ and (c) $2 \times 10^{13}$ ions $/ \mathrm{cm}^{2}$ fluences using a laser with excitation wavelength of $532 \mathrm{~nm}$. The structure of the left side of $\mathrm{G}$ peak for pristine graphene is due to the buffer layer and there is no D peak for pristine graphene. 
The mean distance between defects in graphene $\left(\mathrm{L}_{\mathrm{D}}, \mathrm{nm}\right)$ and the intensity ratio between D and G peaks (I(D)/I(G)) are related by the quantitative formula [60]:

$$
\frac{\mathrm{I}(\mathrm{D})}{\mathrm{I}(\mathrm{G})}=\mathrm{C}_{\mathrm{A}} \frac{\left(r_{A}^{2}-r_{S}^{2}\right)}{\left(r_{A}^{2}-2 r_{S}^{2}\right)}\left[e^{\pi r_{S}^{2} / L_{D}^{2}}-e^{\pi\left(r_{A}^{2}-r_{S}^{2}\right) / L_{D}^{2}}\right]
$$

where $\mathrm{r}_{\mathrm{S}}=1 \mathrm{~nm}, \mathrm{r}_{\mathrm{A}}=3.1 \mathrm{~nm}$, are the radii of "structurally disordered" area and "activated" area around ion induced defects, respectively. Depending on single impact of an ion on graphene, modification in graphene is governed by radii of two circular areas $\mathrm{r}_{\mathrm{A}}$ and $\mathrm{r}_{\mathrm{S}}$ measured from the impact point (with $\mathrm{r}_{\mathrm{A}}>\mathrm{r}_{\mathrm{S}}$ ). The region within $\mathrm{r}_{\mathrm{S}}$ do not contribute much for activation of $\mathrm{D}$ band but the region in between $r_{S}$ and $r_{A}$ contributes strongly for its activation [60]. $C_{A}$ is a constant representing electron-phonon interaction matrix elements and its value at green light excitation estimated from fit to data is $\sim 4.2$ [60]. The relation between defect density $\left(\mathrm{n}_{\mathrm{D}}, \mathrm{cm}^{-2}\right)$ and mean distance between defects $\left(L_{D}, n m\right)$ is given by:

$$
\mathrm{n}_{\mathrm{D}}=10^{14} / \pi L_{D}^{2}
$$

Using equations (3) and (4), we calculated $L_{D}$ and $n_{D}$ of pristine and irradiated graphene on silicon carbide samples at fluences $2 \times 10^{12}$ ions $/ \mathrm{cm}^{2}$ and $2 \times 10^{13} \mathrm{ions} / \mathrm{cm}^{2}$, which are tabulated in Table 1. The values represent average Intensity ratio (I(D)/I(G)) of 10 Raman spectra from the 256 spectra measured during Raman mapping for each pristine and irradiated samples. Table 1 shows that with increase in fluence, intensity ratio (I(D)/I(G)) increases, the mean distance between defects $\left(\mathrm{L}_{D}\right)$ in graphene decreases and the defect density $\left(\mathrm{n}_{\mathrm{D}}\right)$ increases. The calculations also show that this represents stage (i) of defect formation in graphene, which is in confirmation to the observations in Figure 4 where, $\mathrm{I}(\mathrm{D}) / \mathrm{I}(\mathrm{G}) \propto 1 / \mathrm{L}_{\mathrm{D}}{ }^{2}$. With increase in defect density, activated area also increases [60].

Table 1. Summary of Intensity ratio of $D$ and $G$ peak (I(D/I(G)), mean distance between defects in graphene $\left(L_{D}\right)$ and Defect Density $\left(n_{D}\right)$ of Pristine and samples irradiated at fluence $2 \times 10^{12}$ ions $/ \mathrm{cm}^{2}$ and $2 \times 10^{13}$ ions $/ \mathrm{cm}^{2}$ of graphene on silicon carbide

\begin{tabular}{cccc}
\hline Samples & $\mathbf{I}(\mathbf{D}) / \mathbf{I}(\mathbf{G})^{\mathbf{a}}$ & $\mathbf{L}_{\mathbf{D}}(\mathbf{n m})^{\mathbf{a}}$ & $\left.\mathbf{n}_{\mathbf{D}} \times \mathbf{1 0}^{\mathbf{1 0}} \mathbf{( c m}^{-\mathbf{2}}\right)^{\mathbf{a}}$ \\
\hline Pristine & $0.22 \pm 0.007$ & $22.36 \pm 0.95$ & $6.36 \pm 0.81$ \\
$2 \times 10^{12} \mathrm{ions} / \mathrm{cm}^{2}$ & $1.12 \pm 0.07$ & $9.29 \pm 0.27$ & $36.90 \pm 2.07$ \\
$2 \times 10^{13} \mathrm{ions} / \mathrm{cm}^{2}$ & $2.06 \pm 0.09$ & $6.05 \pm 0.09$ & $87.00 \pm 2.59$ \\
\hline
\end{tabular}


${ }^{a}$ Errors represents standard deviation in $\mathrm{I}(\mathrm{D}) / \mathrm{I}(\mathrm{G}), \mathrm{L}_{\mathrm{D}}$ and $\mathrm{n}_{\mathrm{D}}$ values deduced using 10 Raman spectra measured for pristine and irradiated samples.

In the sample irradiated at $2 \times 10^{13}$ ions $/ \mathrm{cm}^{2}$, only about $17 \%$ of monolayer and about 9\% of bilayer is observed due to multiple folding of graphene (Fig. 1(b) and Fig. 3). Table 2 shows percentage of different graphene layers present in pristine and sample irradiated at $2 \times 10^{13}$ ions $/ \mathrm{cm}^{2}$ fluence. Also, reflectance map showed about $64 \%$ bare substrate in sample irradiated at fluence $2 \times 10^{13}$ ions $/ \mathrm{cm}^{2}$. The bare SiC substrate observed in the irradiated samples is due to folding of monolayer graphene into bi-, tri- and multi- layered. This is also supported by Raman mapping results which show presence of bare substrate in irradiated samples but absent in pristine sample and also by AFM images which show folding of graphene (Fig. 3).

Table 2. Number of layers in pristine and irradiated graphene samples at fluences $2 \times 10^{13}$ ions $/ \mathrm{cm}^{2}$ obtained from reflectance mapping.

\begin{tabular}{ccc}
\hline Number of Layers* & \multicolumn{2}{c}{ Samples } \\
\cline { 2 - 3 } & Pristine sample & Sample irradiated at $2 \times 10^{13}$ ions $/ \mathrm{cm}^{2}$ fluence \\
\hline Bare substrate & 0 & 64 \\
1 & 95 & 17 \\
2 & 5 & 9 \\
3 & 0.03 & 4.7 \\
4 & 0.03 & 2.5 \\
5 & 0.04 & 1.4 \\
6 & 0.02 & 0.9 \\
7 & 0.02 & 0.4 \\
8 & 0.01 & 0.2 \\
9 & 0 & 0.06 \\
10 & 0 & 0.12 \\
\hline
\end{tabular}

Fig. 6 (a) shows reflectance map obtained together with Raman mapping of the highestirradiated (at $2 \times 10^{13} \mathrm{ions} / \mathrm{cm}^{2}$ ) sample over a scan area of $4.5 \mu \mathrm{m} \times 4.5 \mu \mathrm{m}$. According to the reflectance map, up to 16 layers have been observed bunching in a hop after irradiation, but the map shown is restricted between 1 and 4 layers for better visibility. It is to be noted that about $56 \%$ of the area is not covered with graphene (according to the reflectance map and the Raman 
spectra) and has only $10.5 \%$ and 5.8\% of ML and bilayer graphene, respectively in this particular area. This hinders conventional plots of parameters obtained from the Raman spectra, but representative Raman spectra of monolayer, multi-layer graphene and bare SiC substrate taken from the same Raman map of the same irradiated sample are displayed in Fig. 6 (b). In the places covered with graphene, Raman mapping shows presence of $\mathrm{D}^{\prime}$ peak along with $\mathrm{D}$ peak. At highest irradiated fluence, the I(D)/ I(D') ratio is 5.22 thereby indicating creation of vacancy defects. It has been previously reported that vacancy defects are created if I(D)/ I(D') is between 3.5 and 7 [59]. Creation of vacancy defects at highest fluence is also corroborated by the $\mathrm{I}(\mathrm{D}) / \mathrm{I}(\mathrm{G})$ ratio ( Fig. 4).

a)
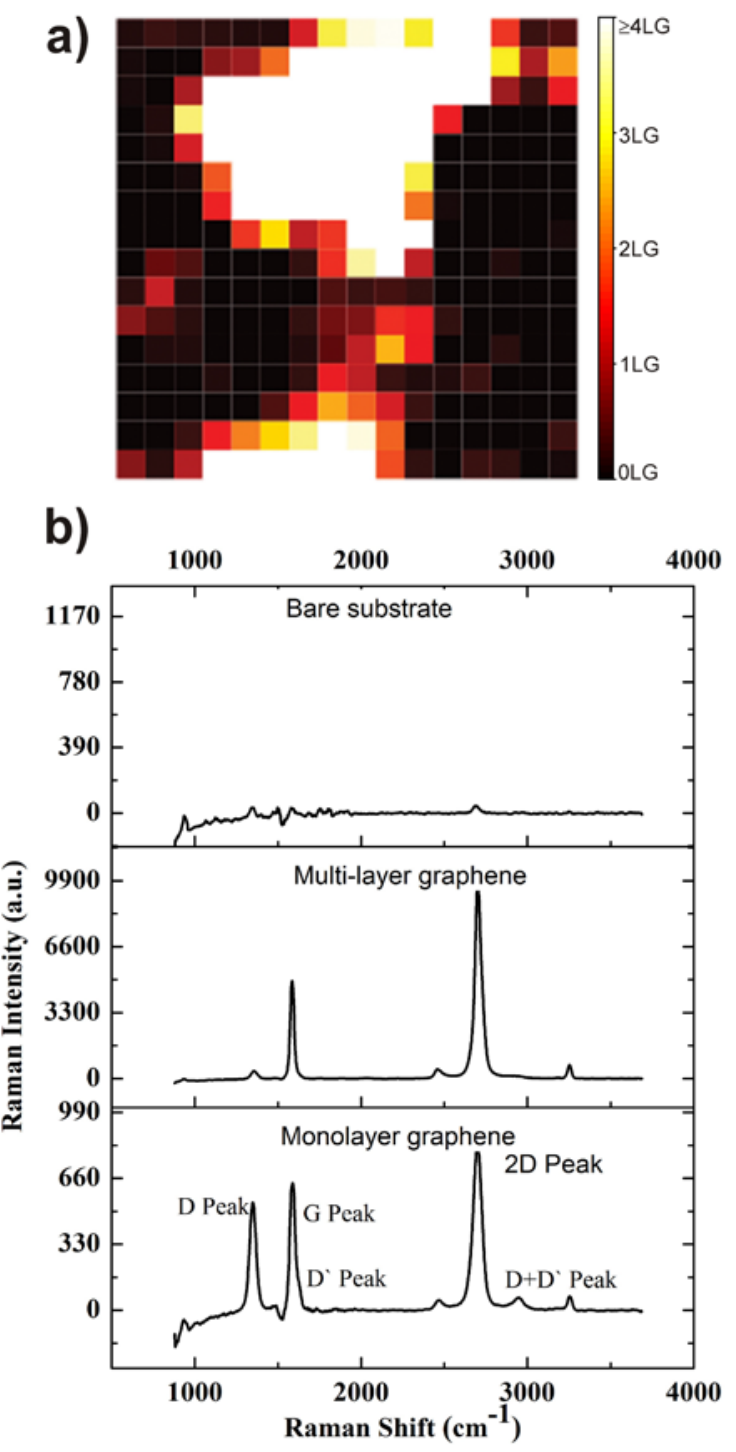
Fig. 6. (a) Reflectance Map of highest irradiated sample scanned over an area of $4.5 \times 4.5 \mu \mathrm{m}$, illustrating formation of islands of multilayer graphene. The map is obtained during the Raman mapping. Up to 16 layers have been observed bunching in a hop after irradiation, but the reflectance map shown is restricted between 1 and 4 layers for better visibility, (b) Raman spectra of bare substrate (SiC), multi-layer graphene formed as a result of irradiation, and monolayer graphene in the sample irradiated at $2 \times 10^{13}$ ions $/ \mathrm{cm}^{2}$ fluence.

Fig. 6(b) shows that monolayer which is still presumably in contact with the substrate shows diminished 2D-peak intensity due to doping [55]. Broadening of 2D peak due to formation of multi-layer graphene can be seen besides reduced 2D, 2D' and G peak intensity in many multilayer spectra (Fig.5(c)). Additionally, we have observed that some multilayer spectrum exhibits some signatures of monolayer graphene, such as $2 \mathrm{D}$ intensity stronger than the $\mathrm{G}$ intensity, as well as symmetric (but significantly broader than true monolayer) single Lorentzian line-shape (Fig. 6(b)). These features are commonly observed in multilayer graphene grown on the C-face (000-1) of the SiC substrate, and are usually associated with presence of multiple layers, which are uncoupled. Also, complete absence of G and 2D peaks in bare substrate spectra show complete removal of graphene from the substrate in that area which is also observed in reflectance map due to multiple folding of graphene. Defects in graphene are used in many applications, and theoretical studies report better gas sensing capabilities in graphene with defects [29, 30]. We explored the gas-sensing capability of these irradiated samples. Fig. 7(a-c) shows the response of sensors fabricated on irradiated epitaxial graphene on $\mathrm{SiC}$ at room temperature towards 10 and $50 \mathrm{ppm} \mathrm{NO}_{2}$ in a background of dry air. Schedin et.al, reported that graphene-based sensors could detect individual events when a gas molecule attaches or detaches from graphene surface [61], and Liu et al showed that since graphene is electronically low noise material, adsorbed molecules change local carrier concentration in graphene in steps of one electron leading to step-like change in resistance [62]. As-grown epitaxial graphene is n-type [63] and $\mathrm{NO}_{2}$ is an oxidizing agent that withdraws electrons from graphene when adsorbed which results in increase in graphene resistance as seen in Fig. 7(a-c). The resistance increase with time due to $\mathrm{NO}_{2}$ exposure and the slope of curve is proportional to the $\mathrm{NO}_{2}$ concentration. We observed that the resistance approached steady state value within 30 minutes thereby showing equilibrium between adsorption and desorption. A previous study showed that desorption occurs for more than $12 \mathrm{~h}$ at room temperature. To reduce desorption time we used UV-LED irradiation and desorption was clearly visible in Fig. 7(a-c) as the 
resistance rapidly recovers upon removal of $\mathrm{NO}_{2}$. However, the initial resistance was not reached in the process of desorption which has also been reported previously and is likely due to strongly adsorbed or chemisorbed $\mathrm{NO}_{2}$ [64]. Fig. 7(a-c) shows the normalized resistance versus time of sensors fabricated on irradiated graphene on $\mathrm{SiC}$ at $6.6 \times 10^{11}, 2 \times 10^{12}$ and $6.6 \times 10^{12}$ ions $/ \mathrm{cm}^{2}$ fluences at room temperature to $\mathrm{NO}_{2}$ gas flow in ppm range for an exposure time of $30 \mathrm{~min}$. The results demonstrate the highest response of the sample at fluence $6.6 \times 10^{11} \mathrm{ions} / \mathrm{cm}^{2}$ in comparison to the other two fluences, $2 \times 10^{12}$ and $6.6 \times 10^{12} \mathrm{ions} / \mathrm{cm}^{2}$. Decreasing response at higher fluences and lower response for pristine sample indicated the existence of an optimal fluence and consequently an optimal amounts of defects which maximize the gas sensing response towards $\mathrm{NO}_{2}$.
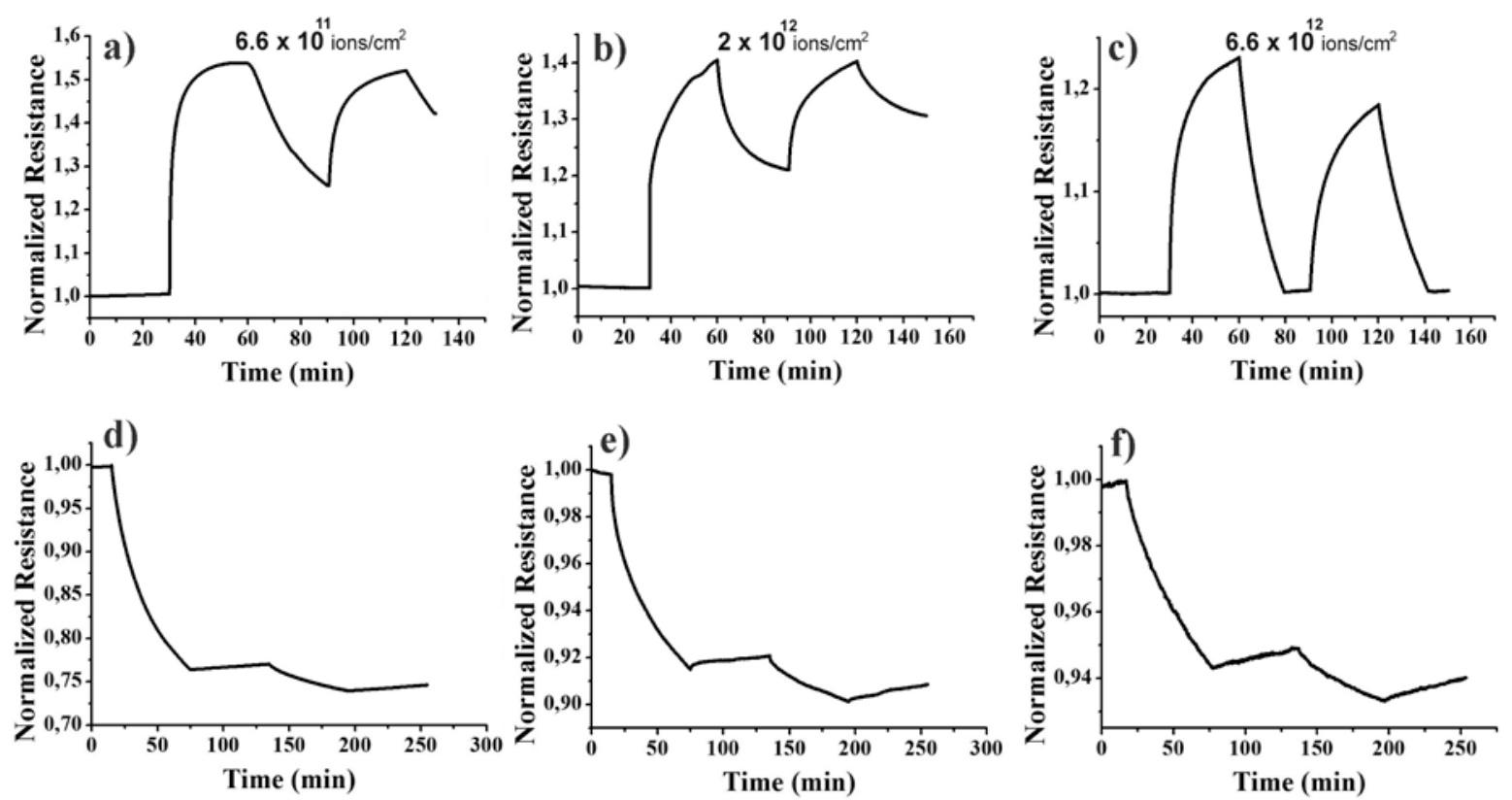

Fig. 7. Response curve of sensor fabricated on irradiated graphene samples at $6.6 \times 10^{11} \mathrm{ions} / \mathrm{cm}^{2}$, $2 \times 10^{12}$ ions $/ \mathrm{cm}^{2}$ and $6.6 \times 10^{12}$ ions $/ \mathrm{cm}^{2}$ fluences, (a-c) exposed at $10 \mathrm{ppm}$ and $50 \mathrm{ppm}$ of $\mathrm{NO}_{2}$ flow. (d-f) exposed at 10ppm and $50 \mathrm{ppm}$ of $\mathrm{NH}_{3}$ flow.

Figure. 7(d-f) show curve of normalized resistance versus time of irradiated graphene samples (at fluences $6.6 \times 10^{11}, 2 \times 10^{12}$ and $6.6 \times 10^{12}$ ions/ $\mathrm{cm}^{2}$ ) at 50 and $10 \mathrm{ppm} \mathrm{NH}_{3}$ flow for an exposure time of 1 hour. $\mathrm{NH}_{3}$ is a strong electron donor for graphene and thereby result in a decrease of the resistance when $\mathrm{NH}_{3}$ is adsorbed on graphene. This is also seen in Fig. 7(d-f) [65]. Our pristine sample does not show any response towards $\mathrm{NH}_{3}$ gas, but all the irradiated samples show such a response. The sample irradiated at $6.6 \times 10^{11} \mathrm{ions} / \mathrm{cm}^{2}$ fluence showed the 
best responses of $4 \%$ and $24 \%$ at for 10 and $50 \mathrm{ppm}$ of $\mathrm{NH}_{3}$ gas, respectively. The sensitivity is defined as:

$$
\mathrm{S}=((\mathrm{R}-\mathrm{Ro}) / \mathrm{Ro})) \times 100 \text {, }
$$

where $\mathrm{R}$ is resistance under test gas exposure and Ro is resistance in carrier gas. At higher than $6.6 \times 10^{11}$ ions $/ \mathrm{cm}^{2}$ fluences the response was observed to decrease with increase in fluence, similar to the response for $\mathrm{NO}_{2}$ gas. A previous study showed that both $\mathrm{sp}^{3}$ and vacancy defects increase the gas-sensing response [66]. In our study, at higher fluences besides vacancy defects, other defects such as graphene folding and presence of higher area of bare substrate might contribute to reduced sensing capabilities. Pearce and co-workers reported that multi-layer graphene on $\mathrm{SiC}$ shows less sensing response compared to monolayer graphene due to higher number of charge carriers in the uppermost layer which are screened from electron donation of $\mathrm{SiC}$ substrate [19]. The reason for decrease in response with increase in fluence after $6.6 \times 10^{11}$ ions $/ \mathrm{cm}^{2}$ fluence is due to more conversion of monolayer graphene to multi-layer which is shown by our Raman and reflectance mapping results. Also, Raman mapping and reflectance mapping results show increased bare $\mathrm{SiC}$ area at higher fluences showing complete detachment of graphene due to multiple folding of graphene layer. Due to increase in bare SiC area with increase in fluence, the effective area of graphene for gas sensing reduces which results in a decreasing sensing capability.

Table 3. Sensitivity of pristine and irradiated samples at fluences $6.6 \times 10^{11}, 2 \times 10^{12}$ and $6.6 \times 10^{12}$ ions/cm ${ }^{2}$ towards the flow of $\mathrm{NO}_{2}$ and $\mathrm{NH}_{3}$ at concentrations 10 and $50 \mathrm{ppm}$. The sensitivity is calculated as percentage of resistance change as explained in text.

\begin{tabular}{lccc}
\hline Gases & Samples & \multicolumn{2}{c}{ Sensitivity ( \%) at concentration } \\
\cline { 3 - 4 } & & $\mathbf{1 0} \mathbf{~ p p m}$ & $\mathbf{5 0} \mathbf{~ p p m}$ \\
\hline $\mathrm{NO}_{2}$ & Pristine & 5.5 & 23.1 \\
& $6.6 \times 10^{11}$ ions $/ \mathrm{cm}^{2}$ & 22.74 & 52.99 \\
& $2 \times 10^{12}$ ions $/ \mathrm{cm}^{2}$ & 19.2 & 40.24 \\
& $6.6 \times 10^{12}$ ions $/ \mathrm{cm}^{2}$ & 18.09 & 22.74 \\
& & \\
& Pristine & 0 & 0 \\
$\mathrm{NH}_{3}$ & $6.6 \times 10^{11}$ ions $/ \mathrm{cm}^{2}$ & 4.45 & 23.89 \\
& $2 \times 10^{12}$ ions $/ \mathrm{cm}^{2}$ & 2.11 & 7.39 \\
& $6.6 \times 10^{12}$ ions $/ \mathrm{cm}^{2}$ & 2.21 & 6.3 \\
\hline
\end{tabular}


Table 3 shows the sensitivity at concentrations 10 and 50 ppm of sensors fabricated at pristine and at varying fluences for $\mathrm{NO}_{2}$ and $\mathrm{NH}_{3}$ gas. It is shown that the sensitivity of pristine sample to $\mathrm{NO}_{2}$ is $5.5 \%$ and $23 \%$ at 10 and $50 \mathrm{ppm}$, respectively. The highest sensitivity of $23 \%$ and $53 \%$ was detected for $\mathrm{NO}_{2}$ gas in sample irradiated at $6.6 \times 10^{11}$ ions $/ \mathrm{cm}^{2}$ fluence. At higher fluences, sensitivity further decreases. The sample irradiated at $6.6 \times 10^{11}$ ions $/ \mathrm{cm}^{2}$ fluence showed also the best sensitivity of $4 \%$ and $24 \%$ at 10 and $50 \mathrm{ppm}$ for $\mathrm{NH}_{3}$ gas. The sensitivity was observed to decrease beyond $6.6 \times 10^{11}$ ions $/ \mathrm{cm}^{2}$ fluence for both test gases. Among the investigated fluences, the defects introduced upon irradiation at fluence $6.6 \times 10^{11} \mathrm{ions} / \mathrm{cm}^{2}$ seem to fit best for gas sensing applications. In our future work, we are planning to explore further gas sensing behavior of irradiated graphene on SiC around this optimized fluence $\left(6.6 \times 10^{11}\right.$ ions $/ \mathrm{cm}^{2}$ ) and also to explore gas sensing capabilities of irradiated samples in lower concentration range (less than $10 \mathrm{ppb}$ ).

\section{Conclusion}

In this study, we irradiated $\mathrm{EG}$ on $\mathrm{SiC}$ substrate with $100 \mathrm{MeV}$ of $\mathrm{Ag}$ ions to investigate structural and morphological changes and investigate their gas sensing capabilities. The AFM study on irradiated graphene layer showed formation of hillocks, wrinkles, and folding of graphene which results in formation of multi-layer graphene due to unzipping of graphene and bunching of graphene fragments. Wrinkles were observed at the highest fluence $\left(2 \times 10^{13}\right.$ ions $/ \mathrm{cm}^{2}$ ). Raman spectroscopy showed increase in defect density with increasing fluence. Additionally, Raman and reflectance mapping showed high percentage of multi-layer graphene formation and bare SiC presence in samples irradiated at higher fluence. Our results also show formation of $\mathrm{sp}^{3}$ defects at low fluence and vacancy defect generation at high fluence on irradiated samples. Gas sensing capabilities of pristine and irradiated samples for $\mathrm{NO}_{2}$ and $\mathrm{NH}_{3}$ gases showed an initial increase in sensing response with maximum at $6.6 \times 10^{11}$ ions $/ \mathrm{cm}^{2}$ fluence, which then decreased upon increasing fluence. This results indicated the existence of an optimal fluence and consequently an optimal amount of defects which maximize the gas sensing response towards $\mathrm{NO}_{2}$ and $\mathrm{NH}_{3}$ gases. The reason for decrease in response with increase in fluence after $6.6 \times 10^{11}$ ions $/ \mathrm{cm}^{2}$ fluence is due to more conversion of monolayer graphene to multi-layer which has less sensing response in comparison with monolayer graphene. This work 
could provide new pathways for increasing sensing capabilities of epitaxial graphene by introducing defects in controlled manner using ion beam technology.

\section{Acknowledgement}

The authors gratefully acknowledge discussions with Rosita Yakimova and the financial support by Graphene Flagship (Contract No. CNECT-ICT-604391) and Swedish Research Council (Project No. 2015-05876).

\section{References}

[1] A.K. Geim, K.S. Novoselov, The rise of graphene, Nature Materials, 6 (2007) 183.

[2] A.K. Geim, P. Kim, Carbon wonderland, Scientific American, 298 (2008) 90.

[3] K.S. Novoselov, A.K. Geim, S.V. Morozov, D. Jiang, M.I. Katsnelson, I.V. Grigorieva, S.V. Dubonos, A.A. Firsov, Two-dimensional gas of massless Dirac fermions in graphene, Nature, 438 (2005) 197.

[4] K.S. Novoselov, A.K. Geim, S.V. Morozov, D. Jiang, Y. Zhang, S.V. Dubonos, I.V. Grigorieva, A.A. Firsov, Electric field in atomically thin carbon films, Science, 306 (2004) 666. [5] Y.H. Wu, T. Yu, Z.X. Shen, Two-dimensional carbon nanostructures: Fundamental properties, synthesis, characterization, and potential applications, Journal of Applied Physics, 108 (2010) 071301.

[6] P.R. Somani, S.P. Somani, M. Umeno, Planer nano-graphenes from camphor by CVD, Chemical Physics Letters, 430 (2006) 56.

[7] C. Berger, Z. Song, T. Li, X. Li, A.Y. Ogbazghi, R. Feng, Z. Dai, N. Alexei, M.E.H. Conrad, P.N. First, W.A. De Heer, Ultrathin epitaxial graphite: 2D electron gas properties and a route toward graphene-based nanoelectronics, Journal of Physical Chemistry B, 108 (2004) 19912.

[8] S.M. Lee, J.H. Kim, J.H. Ahn, Graphene as a flexible electronic material: Mechanical limitations by defect formation and efforts to overcome, Materials Today, 18 (2015) 336.

[9] J.W. Suk, W.H. Lee, J. Lee, H. Chou, R.D. Piner, Y. Hao, D. Akinwande, R.S. Ruoff, Enhancement of the electrical properties of graphene grown by chemical vapor deposition via controlling the effects of polymer residue, Nano Letters, 13 (2013) 1462.

[10] A. Pirkle, J. Chan, A. Venugopal, D. Hinojos, C.W. Magnuson, S. McDonnell, L. Colombo, E.M. Vogel, R.S. Ruoff, R.M. Wallace, The effect of chemical residues on the physical and 
electrical properties of chemical vapor deposited graphene transferred to $\mathrm{SiO}_{2}$, Applied Physics Letters, 99 (2011) 122108.

[11] J. Choi, K.J. Kim, B. Kim, H. Lee, S. Kim, Covalent functionalization of epitaxial graphene by azidotrimethylsilane, Journal of Physical Chemistry C, 113 (2009) 9433.

[12] E. Velez-Fort, C. Mathieu, E. Pallecchi, M. Pigneur, M.G. Silly, R. Belkhou, M. Marangolo, A. Shukla, F. Sirotti, A. Ouerghi, Epitaxial graphene on 4H-SiC(0001) grown under nitrogen flux: Evidence of low nitrogen doping and high charge transfer, ACS Nano, 6 (2012) 10893.

[13] J. Eriksson, D. Puglisi, Y.H. Kang, R. Yakimova, A. Lloyd Spetz, Adjusting the electronic properties and gas reactivity of epitaxial graphene by thin surface metallization, Physica B: Condensed Matter, 439 (2014) 105.

[14] A.L. Hsu, R.J. Koch, M.T. Ong, W. Fang, M. Hofmann, K.K. Kim, T. Seyller, M.S. Dresselhaus, E.J. Reed, J. Kong, T. Palacios, Surface-induced hybridization between graphene and titanium, ACS Nano, 8 (2014) 7704.

[15] J.A. Robinson, M. Labella, K.A. Trumbull, X. Weng, R. Cavelero, T. Daniels, Z. Hughes, M. Hollander, M. Fanton, D. Snyder, Epitaxial graphene materials integration: Effects of dielectric overlayers on structural and electronic properties, ACS Nano, 4 (2010) 2667.

[16] L. Vicarelli, S.J. Heerema, C. Dekker, H.W. Zandbergen, Controlling defects in graphene for optimizing the electrical properties of graphene nanodevices, ACS Nano, 9 (2015) 3428.

[17] K. Yoh, K. Konishi, H. Hibino, Epitaxial graphene FETs with high on/off ratio grown on 4H-SiC, 9th IEEE Conference on Nanotechnology, IEEE NANO 2009, (2009) 334.

[18] Y.M. Lin, C. Dimitrakopoulos, K.A. Jenkins, D.B. Farmer, H.Y. Chiu, A. Grill, P. Avouris, 100-GHz transistors from wafer-scale epitaxial graphene, Science, 327 (2010) 662.

[19] R. Pearce, T. Iakimov, M. Andersson, L. Hultman, A.L. Spetz, R. Yakimova, Epitaxially grown graphene based gas sensors for ultra sensitive $\mathrm{NO}_{2}$ detection, Sensors and Actuators, $\mathrm{B}$ : Chemical, 155 (2011) 451.

[20] C.C. Tang, M.Y. Li, L.J. Li, C.C. Chi, J.C. Chen, Characteristics of a sensitive micro-Hall probe fabricated on chemical vapor deposited graphene over the temperature range from liquidhelium to room temperature, Applied Physics Letters, 99 (2011) 112107.

[21] J.A. Alexander-Webber, A.M.R. Baker, T.J.B.M. Janssen, A. Tzalenchuk, S. Lara-Avila, S. Kubatkin, R. Yakimova, B.A. Piot, D.K. Maude, R.J. Nicholas, Phase space for the breakdown of the quantum hall effect in epitaxial graphene, Physical Review Letters, 111 (2013) 096601. 
[22] P. Willke, J.A. Amani, S. Thakur, S. Weikert, T. Druga, K. Maiti, H. Hofsäss, M. Wenderoth, Short-range ordering of ion-implanted nitrogen atoms in SiC-graphene, Applied Physics Letters, 105 (2014) 111605.

[23] D.K. Avasthi, Modification and characterisation of materials by swift heavy ions, Defence Science Journal, 59 (2009) 401.

[24] S. Kumar, A. Tripathi, F. Singh, S.A. Khan, V. Baranwal, D.K. Avasthi, Purification/annealing of graphene with 100-MeV Ag ion irradiation, Nanoscale Research Letters, 9 (2014) 126.

[25] O. Ochedowski, S. Akcöltekin, B. Ban-D'Etat, H. Lebius, M. Schleberger, Detecting swift heavy ion irradiation effects with graphene, Nuclear Instruments and Methods in Physics Research, Section B: Beam Interactions with Materials and Atoms, 314 (2013) 18.

[26] S. Akcöltekin, H. Bukowska, T. Peters, O. Osmani, I. Monnet, I. Alzaher, B.B. D'Etat, H. Lebius, M. Schleberger, Unzipping and folding of graphene by swift heavy ions, Applied Physics Letters, 98 (2011) 103103.

[27] J. Eriksson, D. Puglisi, C. Strandqvist, R. Gunnarsson, S. Ekeroth, I.G. Ivanov, U. Helmersson, K. Uvdal, R. Yakimova, A.L. Spetz, Modified Epitaxial Graphene on SiC for Extremely Sensitive and Selective Gas Sensors, Materials Science Forum, 858 (2016), pp. 11451148.

[28] H. Li, M. Huang, G. Cao, Markedly different adsorption behaviors of gas molecules on defective monolayer MoS2: A first-principles study, Physical Chemistry Chemical Physics, 18 (2016) 15110.

[29] Y. Jiang, S. Yang, S. Li, W. Liu, Y. Zhao, Highly Sensitive CO Gas Sensor from Defective Graphene: Role of van der Waals Interactions, Journal of Nanomaterials, 2015 (2015) 504103. [30] Y.H. Zhang, Y.B. Chen, K.G. Zhou, C.H. Liu, J. Zeng, H.L. Zhang, Y. Peng, Improving gas sensing properties of graphene by introducing dopants and defects: A first-principles study, Nanotechnology, 20 (2009) 185504.

[31] G.R. Yazdi, R. Vasiliauskas, T. Iakimov, A. Zakharov, M. Syväjärvi, R. Yakimova, Growth of large area monolayer graphene on $3 \mathrm{C}$-SiC and a comparison with other SiC polytypes, Carbon, 57 (2013) 477.

[32] J.F. Ziegler, M.D. Ziegler, J.P. Biersack, SRIM - The stopping and range of ions in matter (2010), Nuclear Instruments and Methods in Physics Research, Section B: Beam Interactions with Materials and Atoms, 268 (2010) 1818.

[33] I.G. Ivanov, J.U. Hassan, T. Iakimov, A.A. Zakharov, R. Yakimova, E. Janzén, Layernumber determination in graphene on SiC by reflectance mapping, Carbon, 77 (2014) 492. 
[34] M.L. Bolen, S.E. Harrison, L.B. Biedermann, M.A. Capano, Graphene formation mechanisms on 4H-SiC(0001), Physical Review B, 80 (2009) 115433.

[35] H. Hibino, H. Kageshima, M. Nagase, Epitaxial few-layer graphene: towards single crystal growth, Journal of Physics D: Applied Physics, 43 (2010) 374005.

[36] S. Zhao, J. Xue, Modification of graphene supported on SiO2 substrate with swift heavy ions from atomistic simulation point, Carbon, 93 (2015) 169.

[37] S. Bouffard, J. Cousty, Y. Pennec, F. Thibaudau, STM and AFM observations of latent tracks, Radiation Effects and Defects in Solids, 126 (1993) 225.

[38] R. Neumann, Scanning probe microscopy of ion-irradiated materials, Nuclear Instruments and Methods in Physics Research, Section B: Beam Interactions with Materials and Atoms, 151 (1999) 42.

[39] N. Khalfaoui, M. Görlich, C. Müller, M. Schleberger, H. Lebius, Latent tracks in CaF2 studied with atomic force microscopy in air and in vacuum, Nuclear Instruments and Methods in Physics Research, Section B: Beam Interactions with Materials and Atoms, 245 (2006) 246. [40] M. Toulemonde, C. Dufour, E. Paumier, The ion-matter interaction with swift heavy ions in the light of inelastic thermal spike model, Acta Physica Polonica A, 109 (2006) 311.

[41] E. Akcöltekin, T. Peters, R. Meyer, A. Duvenbeck, M. Klusmann, I. Monnet, H. Lebius, M. Schleberger, Creation of multiple nanodots by single ions, Nature Nanotechnology, 2 (2007) 290.

[42] P.K. Sharma, V. Sharma, R.S. Rajaura, S. Srivastava, S.S. Sharma, M. Singh, Y.K. Vijay, SHI induced defects in chemically synthesized graphene oxide for hydrogen storage applications, AIP Conference Proceedings, 1728 (2016) 020531.

[43] B. Lalmi, J.C. Girard, E. Pallecchi, M. Silly, C. David, S. Latil, F. Sirotti, A. Ouerghi, Flower-shaped domains and wrinkles in trilayer epitaxial graphene on silicon carbide, Scientific Reports, 4 (2014) 4066.

[44] E. Loginova, S. Nie, K. Thürmer, N.C. Bartelt, K.F. McCarty, Defects of graphene on Ir(111): Rotational domains and ridges, Physical Review B - Condensed Matter and Materials Physics, 80 (2009) 085430.

[45] B.W. Smith, D.E. Luzzi, Electron irradiation effects in single wall carbon nanotubes, Journal of Applied Physics, 90 (2001) 3509.

[46] M.T. Lusk, L.D. Carr, Nanoengineering defect structures on graphene, Physical Review Letters, 100 (2008) 175503.

[47] L.M. Malard, M.A. Pimenta, G. Dresselhaus, M.S. Dresselhaus, Raman spectroscopy in graphene, Physics Reports, 473 (2009) 51. 
[48] A.C. Ferrari, Raman spectroscopy of graphene and graphite: Disorder, electron-phonon coupling, doping and nonadiabatic effects, Solid State Communications, 143 (2007) 47.

[49] F. Tuinstra, J.L. Koenig, Raman Spectrum of Graphite, The Journal of Chemical Physics, 53 (1970) 1126.

[50] R.J. Nemanich, S.A. Solin, First- and second-order Raman scattering from finite-size crystals of graphite, Physical Review B, 20 (1979) 392.

[51] A.C. Ferrari, J.C. Meyer, V. Scardaci, C. Casiraghi, M. Lazzeri, F. Mauri, S. Piscanec, D. Jiang, K.S. Novoselov, S. Roth, A.K. Geim, Raman spectrum of graphene and graphene layers, Physical Review Letters, 97 (2006) 187401.

[52] L.G. Cançado, A. Jorio, E.H.M. Ferreira, F. Stavale, C.A. Achete, R.B. Capaz, M.V.O. Moutinho, A. Lombardo, T.S. Kulmala, A.C. Ferrari, Quantifying defects in graphene via Raman spectroscopy at different excitation energies, Nano Letters, 11 (2011) 3190.

[53] J.S. Park, A. Reina, R. Saito, J. Kong, G. Dresselhaus, M.S. Dresselhaus, G' band Raman spectra of single, double and triple layer graphene, Carbon, 47 (2009) 1303-1310.

[54] O. Ochedowski, B. Kleine Bussmann, B. Ban Detat, H. Lebius, M. Schleberger, Manipulation of the graphene surface potential by ion irradiation, Applied Physics Letters, 102 (2013) 153103.

[55] Z.H. Ni, T. Yu, Z.Q. Luo, Y.Y. Wang, L. Liu, C.P. Wong, J. Miao, W. Huang, Z.X. Shen, Probing charged impurities in suspended graphene using raman spectroscopy, ACS Nano, 3 (2009) 569-574.

[56] J. Röhrl, M. Hundhausen, F. Speck, T. Seyller, Strain and charge in epitaxial graphene on silicon carbide studied by raman spectroscopy, in: Materials Science Forum, 645-648 (2010) 603.

[57] A. Zandiatashbar, G.H. Lee, S.J. An, S. Lee, N. Mathew, M. Terrones, T. Hayashi, C.R. Picu, J. Hone, N. Koratkar, Effect of defects on the intrinsic strength and stiffness of graphene, Nature Communications, 5 (2014) 3186.

[58] D.C. Elias, R.R. Nair, T.M.G. Mohiuddin, S.V. Morozov, P. Blake, M.P. Halsall, A.C. Ferrari, D.W. Boukhvalov, M.I. Katsnelson, A.K. Geim, K.S. Novoselov, Control of graphene's properties by reversible hydrogenation: Evidence for graphane, Science, 323 (2009) 610.

[59] A. Eckmann, A. Felten, A. Mishchenko, L. Britnell, R. Krupke, K.S. Novoselov, C. Casiraghi, Probing the nature of defects in graphene by Raman spectroscopy, Nano Letters, 12 (2012) 3925. 
[60] M. M. Lucchese, F. Stavale, E. H.M. Ferreira, C. Vilani, M.V.O. Moutinho, R.B. Capaz, C.A. Achete, A. Jorio, Quantifying ion-induced defects and Raman relaxation length in graphene, Carbon, 48 (2010) 1592.

[61] F. Schedin, A.K. Geim, S.V. Morozov, E.W. Hill, P. Blake, M.I. Katsnelson, K.S. Novoselov, Detection of individual gas molecules adsorbed on graphene, Nature Materials, 6 (2007) 652.

[62] G. Liu, W. Stillman, S. Rumyantsev, Q. Shao, M. Shur, A.A. Balandin, Low-frequency electronic noise in the double-gate single-layer graphene transistors, Applied Physics Letters, 95 (2009) 033103.

[63] F. Varchon, R. Feng, J. Hass, X. Li, B.N. Nguyen, C. Naud, P. Mallet, J.Y. Veuillen, C. Berger, E.H. Conrad, L. Magaud, Electronic structure of epitaxial graphene layers on SiC: Effect of the substrate, Physical Review Letters, 99 (2007) 126805.

[64] T. Ueda, M.M.H. Bhuiyan, H. Norimatsu, S. Katsuki, T. Ikegami, F. Mitsugi, Development of carbon nanotube-based gas sensors for NOx gas detection working at low temperature, Physica E: Low-Dimensional Systems and Nanostructures, 40 (2008) 2272.

[65] O. Leenaerts, B. Partoens, F.M. Peeters, Adsorption of $\mathrm{H}_{2} \mathrm{O}, \mathrm{NH} 3, \mathrm{CO}, \mathrm{NO}_{2}$, and $\mathrm{NO}$ on graphene: A first-principles study, Physical Review B - Condensed Matter and Materials Physics, 77 (2008) 125416.

[66] G. Lee, G. Yang, A. Cho, J.W. Han, J. Kim, Defect-engineered graphene chemical sensors with ultrahigh sensitivity, Physical Chemistry Chemical Physics, 18 (2016) 14198. 